\title{
EXTENDED FAILURE MODELS FOR GLOBAL AND LOCAL ANALYSES OF COMPOSITE AEROSTRUCTURES
}

\author{
Giuseppe Corrado $^{1,2 *}$, Albertino Arteiro ${ }^{1}$, José Reinoso ${ }^{3}$, Florian Glock ${ }^{2}$ and Fernass \\ Daoud $^{2}$ \\ ${ }^{1}$ Department of Mechanical Engineering, Faculty of Engineering, University of Porto, Porto, Portugal \\ ${ }^{2}$ Airbus Defence and Space GmbH, Manching, Germany \\ ${ }^{3}$ Group of Elasticity and Strength of Materials, University of Seville, Seville, Spain \\ * giuseppe.corrado@airbus.com
}

Detailed numerical models for large aerospace products result in prohibitive computational costs with the current state of technology. Therefore, to overcome the need of high-fidelity models involving millions of degrees of freedom, efficient modelling strategies have been recently proposed, such as global/local approaches. In this framework, "hot-spot" identification analysis can be used to highlight the most critical structural details where a local model would have a crucial role in capturing more accurately the structural behavior. With the obtained indications, an efficient global/local modelling strategy can be implemented to address the structural response of composite structures.

This paper deals with the implementation of an advanced failure model, based on invariant structural tensors, to predict the inelastic deformation and fracture of laminated composites. A 3D invariant-based failure theory [1] was implemented in a user subroutine to perform "hotspot" first-ply failure (FPF) analysis and predict the onset of intralaminar damage, identifying the critical failure mode. This approach was also validated against challenging experimental results from the literature involving open-hole plates subjected to quasi-pure bending loadings. Thus, the described method proved to be a very robust tool, giving reliable and fast indications suitable for conceptual and preliminary design of composite structures.

Although "hot-spot" FPF analysis can be useful in guiding preliminary design and identifying the most critical areas, it is not sufficient to predict the ultimate load of composite laminates, since failure of these materials is governed by a progressive evolution of damage, which must be addressed for critical areas. Hence, to predict the inelastic deformation up to ultimate failure in local models, these 3D invariant-based criteria are coupled with a smeared crack model that accounts for the kinematics of matrix crack and kink band formation during damage propagation. To predict delamination, cohesive elements are used at the interfaces between layers with different orientation. Validation is performed against experimental results at the coupon and sub-component level using current aerospace-grade composite systems, revealing the accuracy of the proposed damage model and its suitability for local detailed analysis of composite aerostructures.

Acknowledgements. This work was supported by the H2020 Marie Sklodowska-Curie European Industrial Doctorate OptiMACS (Grant 764650).

\section{References}

[1] P.P. Camanho, A. Arteiro, A.R. Melro, G. Catalanotti and M. Vogler (2015) Threedimensional invariant-based failure criteria for fibre-reinforced composites. International Journal of Solids and Structures, 55, 92-107. 